PROCEEDINGS OF THE

AMERICAN MATHEMATICAL SOCIETY

Volume 128, Number 4, Pages 1167-1176

S 0002-9939(99)05341-1

Article electronically published on August 5, 1999

\title{
THE INDEX OF TRIANGULAR OPERATOR MATRICES
}

\author{
K.-H. FÖRSTER AND B. NAGY \\ (Communicated by David R. Larson)
}

\begin{abstract}
For any triangular operator matrix acting in a direct sum of complex Banach spaces, the order of a pole of the resolvent (i.e. the index) is determined as a function of the coefficients in the Laurent series for all the (resolvents of the) operators on the diagonal and of the operators below the diagonal. This result is then applied to the case of certain nonnegative operators in Banach lattices. We show how simply these results imply the Rothblum Index Theorem (1975) for nonnegative matrices. Finally, examples for calculating the index are presented.
\end{abstract}

\section{INTRODUCTION}

The index or, in other words, the order of a complex number $z_{0}$ as a pole of the resolvent of a triangular operator matrix as a function of the orders of the poles for the corresponding operators on the diagonal has recently been studied by a number of authors with remarkable results. One of the most significant results of this type is the Rothblum Index Theorem [ $\mathbb{R}$, Theorem 3.1(2)] in the case of a nonnegative matrix (after bringing it by a permutation similarity to a Frobenius normal form) and of the spectral radius (as a pole of the resolvent). Problems of this type in the case of a nonnegative operator with compact powers (and with a positive spectral radius) in a Banach lattice with order continuous norm have been studied by JangLewis and Victory JLV Theorem V.1(2)], where the method of the solution was different. The more general case of a not necessarily nonnegative operator and of a general $z_{0} \in \mathbf{C}$ was studied in a finite dimensional space, e.g. by Meyer and Rose [MR], by Friedland and Hershkowitz [FH] and by Hershkowitz, Rothblum and Schneider [HRS], and a complete solution for the case of two matrices on the diagonal was obtained by Bru, Climent and Neumann [BCN].

The purpose of this note is twofold. First we show in Theorem 1 that the general case of an infinite dimensional Banach space and bounded linear operators in the (lower) triangular operator matrix (having $z_{0}$ at most as a pole for the operators on the diagonal) can be solved by modifying the graph theoretic method applied by Rothblum $[\mathrm{R}$. Then we show in Theorem 2 how this modified method applies to the case of certain nonnegative operators (matrices) and of the spectral radius. The straightforward Corollaries 1 and 2 to Theorems 1 and 2 will give a different

Received by the editors October 10, 1997 and, in revised form, June 17, 1998.

1991 Mathematics Subject Classification. Primary 47A10, 47B65; Secondary 15A18, 15A48.

Key words and phrases. Triangular operator matrix, order of pole, Banach lattice, nonnegative operator and matrix, Index Theorem.

The second author was supported by the Hungarian NSF Grant (OTKA No. T-016925).

(C)2000 American Mathematical Society 
equivalent expression for the index in the setting of Jang-Lewis and Victory [JLV], and will reproduce the Rothblum Index Theorem $[\mathrm{R}$, respectively. We feel that this method of proof is even simpler than the original proof of the Index Theorem, makes apparent the significance of nonnegativity, and is completely different from the method of [BCN]. Finally, in Section 3 we shall present a number of examples which will show the effective applicability of our general results in the cases both of nonnegative and of not necessarily nonnegative triangular operator matrices.

For the needed preliminaries on nonnegative operators in Banach lattices we refer to the corresponding places in the monographs by Aliprantis and Burkinshaw ([AB]) and by Schaefer ([SBL, $[\mathrm{ST}])$.

\section{The RESULts}

Assume that the Banach space $X$ is the direct sum of the Banach spaces $X_{1}, \ldots$, $X_{m}$, and that the continuous linear operator $A: X \rightarrow X$ has a lower triangular block form with respect to the direct sum above. Thus the operator $A$ is represented by the operator matrix

$$
A=\left(\begin{array}{ccccc}
A_{1} & 0 & 0 & \ldots & 0 \\
A_{21} & A_{2} & 0 & \ldots & 0 \\
A_{31} & A_{32} & A_{3} & \ldots & 0 \\
\ldots & \ldots & & & \\
A_{m 1} & A_{m 2} & A_{m 3} & \ldots & A_{m}
\end{array}\right) .
$$

Assume further that the point $z_{0} \in \mathbf{C}$ is a pole for all the resolvents of the operators $A_{1}, \ldots, A_{m}$ of corresponding orders $p_{1}, \ldots, p_{m} \geq 0$. Observe that this implies that $z_{0}$ is a pole of the resolvent of $A$.

In a similar spirit as in some matrix problems of combinatorial nature we shall call the set $C:=\{1, \ldots, m\}$ the set of all classes for $A$, and an ordered set $\left\{k_{1}, \ldots, k_{s}\right\} \subset C$ a chain of classes either if it is a singleton, or if

$$
k_{1}>k_{2}>\cdots>k_{s}, \quad A_{k_{1} k_{2}} \neq 0, \ldots, A_{k_{s-1} k_{s}} \neq 0 .
$$

By assumption, every resolvent $R\left(z, A_{k}\right)=\left(z-A_{k}\right)^{-1}$ has the Laurent expansion in a deleted neighbourhood of $z_{0}$ :

$$
R\left(z, A_{k}\right)=\sum_{r=-p_{k}}^{\infty}\left(z-z_{0}\right)^{r} C_{k}^{(r)} .
$$

For $k_{1}>k_{s}$ we shall call a product of the form

$$
C_{k_{1}}^{\left(q_{1}\right)} A_{k_{1} k_{2}} C_{k_{2}}^{\left(q_{2}\right)} \cdots A_{k_{s-1} k_{s}} C_{k_{s}}^{\left(q_{s}\right)}
$$

a $\left(k_{1}, k_{s}\right)$-weighted chain of length $s$ with weight $w:=q_{1}+\cdots+q_{s}$. For $s=1$ and $i:=k_{1}=k_{s}$ we shall apply the same terminology to the operator $C_{i}^{(w)}$. Further, for a fixed pair $\left(k_{1}, k_{s}\right)$ and a fixed weight $w$ we shall call the $\left(k_{1}, k_{s}, w\right)$-sum the sum of all $\left(k_{1}, k_{s}\right)$-weighted chains of any lengths with the fixed weight $w$.

With this terminology we have the following basic

Theorem 1. Under the above conditions $z_{0}$ is a pole of exact order $p$ for the resolvent $R(z, A)$, where

$$
\max \left\{p_{1}, \ldots, p_{m}\right\} \leq p \leq p_{1}+\cdots+p_{m},
$$


if and only if there is a pair $\left(k_{1}, k_{s}\right)$ with $k_{1} \geq k_{s}$ such that the $\left(k_{1}, k_{s},-p\right)$-sum is not the operator 0 , and for every pair $(i, j)$ satisfying $m \geq i \geq j \geq 1$, the $(i, j,-p-1)$-sum is the operator 0 .

Proof. It is well-known that the lower triangular block form of the operator $A$ implies that its spectrum $\sigma(A)$ is contained in $\bigcup_{k=1}^{m} \sigma\left(A_{k}\right)$. Further, it can be seen by induction that for $z \notin \bigcup_{k=1}^{m} \sigma\left(A_{k}\right)$ the operator $R(z, A)$ also has a lower triangular matrix form with the following operator entry in the block row $i$ and block column $j$ for the pair satisfying $m \geq i \geq j \geq 1$ :

$$
\begin{gathered}
R(z, A)_{i j}=R\left(z, A_{i}\right) \quad \text { for } \quad i=j, \\
R(z, A)_{i j}=\sum_{s=2}^{i-j+1} \sum R\left(z, A_{i}\right) A_{i k_{2}} R\left(z, A_{k_{2}}\right) A_{k_{2} k_{3}} \cdots A_{k_{s-1} j} R\left(z, A_{j}\right) \quad \text { for } \quad i>j,
\end{gathered}
$$

where the second summation is over all $(s-2)$-tuples $\left(k_{2}, k_{3}, \ldots, k_{s-1}\right)$ satisfying $i>k_{2}>\cdots>k_{s-1}>j$. Observe that the second sum above for the value $s=2$ reduces simply to $R\left(z, A_{i}\right) A_{i j} R\left(z, A_{j}\right)$.

From the assumptions it follows that in a deleted neighbourhood of $z_{0}$ the Laurent expansions of all the resolvents $R\left(z, A_{k}\right)$ converge. By forming their suitable products and reordering them, from the formulae for the entries of the operator $R(z, A)$ we obtain for $i>j$

$$
R(z, A)_{i j}=\sum_{w=-p_{1}-\cdots-p_{m}}^{\infty}\left(z-z_{0}\right)^{w} \sum C_{i}^{\left(q_{1}\right)} A_{i k_{2}} C_{k_{2}}^{\left(q_{2}\right)} \cdots A_{k_{s-1} j} C_{j}^{\left(q_{s}\right)},
$$

where the second (finite) sum extends to all $(i, j)$-weighted chains of arbitrary admissible lengths with the weight $q_{1}+\cdots+q_{s}=w$, i.e. is exactly the $(i, j, w)$-sum defined above. It is immediate from the definition that for $i=j$ the corresponding coefficient in the series

$$
R(z, A)_{i i}=R\left(z, A_{i}\right)=\sum_{w=-p_{i}}^{\infty}\left(z-z_{0}\right)^{w} C_{i}^{(w)}
$$

is exactly the $(i, i, w)$-sum.

For the operator matrix $A$ the number $z_{0}$ is a pole of exact order $p$ if and only if $z_{0}$ is a pole of exact order $p$ for some entry of the resolvent operator $R(z, A)$ and not a pole of higher order or an essential singularity for any entry. By the above representation of the entries, this holds exactly when the statement in the Theorem holds.

Finally observe that though the estimate for the order $p$ of the pole $z_{0}$ is essentially known, it follows simply from the proved part of the Theorem.

Remark. Observe that the above proof also shows the validity of a more exact estimate for $p$, which is called the Index Theorem (for finite dimensional spaces) in [FH] and [HRS]:

$$
p \leq \max \left(p_{k_{1}}+\cdots+p_{k_{r}}\right),
$$

where the maximum is taken over all chains $\left\{k_{1}, \ldots, k_{r}\right\}$ in $C$.

We shall now apply Theorem 1 for the description of the order of the spectral radius as a pole of certain nonnegative (reducible) linear operators in certain Banach lattices considered by Jang-Lewis and Victory in [JLV]. Our characterization of the 
order will be different from (but, naturally, equivalent with) theirs, and will be in the same spirit as the corresponding celebrated result for nonnegative matrices by Rothblum $[\underline{\mathrm{R}}$.

First we cite the relevant decomposition result from [JLV] p. 64].

Theorem ([JLV]). Let $X$ be a Banach lattice with order continuous norm and $T$ be a nonnegative (reducible) eventually compact operator with positive spectral radius $R$, which is a pole of exact order $d-1$ of the resolvent. Then we can decompose $X$ as a finite orthogonal sum of projection bands such that the matrix of the operator $T$ has a lower block triangular form with respect to this decomposition. The first $d-1$ of the $d$ diagonal blocks have the structure

$$
T_{k}=\left(\begin{array}{ccccc}
T_{10}^{(k)} & 0 & 0 & \ldots & 0 \\
x & T_{11}^{(k)} & 0 & \ldots & 0 \\
\vdots & 0 & \ddots & T_{1, n(k)-1}^{(k)} & 0 \\
x & 0 & 0 & \ldots & T_{1, n(k)}^{(k)}
\end{array}\right) \quad(k=1, \ldots, d-1)
$$

where all the operators are nonnegative, and the operators denoted by $x$ may also be (different and) nonzero. Further, the operators $T_{11}^{(k)}, \ldots, T_{1, n(k)}^{(k)}$ on the diagonal are irreducible, and for the spectral radii we have

$$
r\left(T_{10}^{(k)}\right)<R, \quad r\left(T_{1 h}^{(k)}\right)=R \quad(k=1, \ldots, d-1, h=1, \ldots, n(k)) .
$$

Finally, the nonnegative operator $T_{d}$ is not necessarily irreducible, and $r\left(T_{d}\right)<R$.

Now we apply again for the operator $A:=T$ our standard notation from the beginning of the section in such a form that the operators $A_{k}$ will be the (inner) diagonal operators. Since some of the operators $T_{10}^{(k)}$ may be missing, we shall have $m \leq n(1)+1+\cdots+n(d-1)+1+1$. Observe that under these conditions every operator $A_{k}(k=1, \ldots, m)$ either is irreducible with $r\left(A_{k}\right)=R$, or satisfies $r\left(A_{k}\right)<R$. By [AB pp. 277-278], all operators on the diagonal have compact powers; therefore their spectral radii, provided they are positive, are poles of their resolvents. By [ST, App. 3.2], the respective orders of the pole $R$ are 1. Hence for any weighted chain for $A$ and $z_{0}:=R$ with the classes $k_{1}>\cdots>k_{s}$, the lowest possible weight $w$ obtains exactly when all the numbers $q_{1}, \ldots, q_{s}$ are equal to the negatives of the corresponding pole orders, and then $q_{h}=-1$ or 0 for every $h$. If $s=1$, then again $q_{1}=-1$ or 0 , depending on the order of the pole $R$. From Theorem 1 we obtain now the following

Corollary 1. Let $X$ a Banach lattice with order continuous norm and $A$ a nonnegative eventually compact operator with positive spectral radius $R$, and consider the standard decomposition of $X$ and the corresponding lower triangular form of the operator matrix of $A$ discussed above. Then the order of the pole $z_{0}:=R$ for the (resolvent of the) operator $A$ (i.e. the Riesz index of $R$ for $A$ in [JLV, Theorem V.1(2)]) is the negative of the lowest possible weight for an arbitrary weighted chain for $A$ and $z_{0}=R$ of any admissible length. This order is equal to the number of the basic classes (i.e. classes $j$ for which $r\left(A_{j}\right)=R$ ) in such a chain.

Proof. From the position of the zero operators in each block $T_{k}$ we see that the lowest possible weight $w$ satisfies $w \geq-d+1$. The operators denoted by the letters $C$ in (2) are nonnegative in any weighted chain with the lowest weight $w$. Indeed, either they are of the form $\left(R-A_{j}\right)^{-1}$, where the nonnegative operator $A_{j}$ has 
spectral radius $<R$, or they are the residuum $P_{k}$ at $R$ of the resolvent $\left(z-A_{k}\right)^{-1}$ for some nonnegative irreducible operator $A_{k}$ with spectral radius $R$, which is a pole of the resolvent of order 1 and rank 1 (see [ST, App. 3.2]). In either case they are nonnegative, and then, by assumption, the corresponding weighted chain (2) is nonnegative. If every such chain is the 0 operator, then, by Theorem 1 , the order of the pole $R$ for $A$ is less than $d-1$, a contradiction. This proves both claims in the Corollary.

Theorem 2. Assume that the Banach lattice $X$ is the direct sum of the Banach lattices $X_{1}, \ldots, X_{m}$, the continuous linear operator $A: X \rightarrow X$ has the operator matrix representation (1) with respect to this direct sum, the operator entries are all nonnegative (between the corresponding spaces), and the diagonal entries $A_{1}, \ldots, A_{m-1}$ are irreducible operators (in their respective spaces) such that

$$
R:=\max _{1 \leq j \leq m-1} r\left(A_{j}\right)>0,
$$

where each spectral radius $r\left(A_{j}\right)$ equaling $R$ is a pole of the resolvent $\left(z-A_{j}\right)^{-1}$, and $r\left(A_{m}\right)<R$. Then every weighted chain (for $A$ and $z_{0}:=R$ ) with the lowest possible weight is a nonnegative and nonzero operator.

Proof. First consider the case when the chain is a singleton, say $\{j\}$. If $r\left(A_{j}\right)=R$, then the order of this pole is 1 , and the corresponding residue (projection) $P$ has the form $P=\beta \otimes u$ for some quasi-interior point $u$ in $X_{j+}$ and for some $\beta$ in the dual space $X_{j}^{\prime}$, by [ST, App. 3.2]. The range of the adjoint projection $P^{\prime}$ is the annihilator of $\operatorname{ker}(P)$, i.e. the annihilator of $\operatorname{ker}(\beta)$. Hence $\beta$ is in $\operatorname{ker}\left(A_{j}^{\prime}-R\right)$. Applying [ST, App. 3.2] again, the functional $\beta$ is strictly positive. Hence $P$ is strictly positive. Therefore in the standard notation for a weighted chain $C_{j}^{(-1)}$ is strictly positive and maps $X_{j+} \backslash\{0\}$ into the set $Q$ of all quasi-interior points in $X_{j+}$.

On the other hand, if $r\left(A_{j}\right)<R$, then the resolvent operator $\left(R-A_{j}\right)^{-1}$ is nonnegative and invertible, hence nonzero. Further, if $A_{j}$ is irreducible (which certainly holds for $j=1, \ldots, m-1$ ), we can prove and shall need more. By the definition of irreducibility (cf. [ST App. 3]), the operator

$$
A_{j}\left(R-A_{j}\right)^{-1}=-I+R\left(R-A_{j}\right)^{-1}
$$

maps $X_{j+} \backslash\{0\}$ into the set $Q$. Hence $\left(R-A_{j}\right)^{-1}$ also maps $X_{j+} \backslash\{0\}$ into $Q$. Therefore in the standard notation for a weighted chain $C_{j}^{(0)}$ is nonnegative, invertible, and if $1 \leq j \leq m-1$, then it maps $X_{j+} \backslash\{0\}$ into $Q$.

Now consider the case of a weighted chain for which $k_{1}>k_{s}$, and the lowest possible weight $w=q_{1}+\cdots+q_{s}$ is achieved, so that $q_{j}=-1$ if $r\left(A_{k_{j}}\right)=R$, and $q_{j}=0$ otherwise $(j=1, \ldots, s)$. From the above considerations it is clear that the product is a nonnegative operator. We still have to show that it is nonzero.

Observe first that for any nonnegative, nonzero operator $T$ between two Banach lattices and for any quasi-interior nonnegative element $y$ from the domain, the nonnegative element $T y$ is necessarily nonzero. Indeed, $T y=0$ would imply for the corresponding principal ideal $E_{y}$ (cf. [SBL p. 57]) that

$$
T E_{y}=\bigcup_{n=1}^{\infty} n T[-y, y]=\{0\} .
$$


Since $y$ is a quasi-interior point, $E_{y}$ is dense in the domain space. This would imply $T=0$, a contradiction. Now let $x$ be any nonnegative nonzero element in the corresponding domain space and consider

$$
C_{k_{1}}^{\left(q_{1}\right)} A_{k_{1} k_{2}} C_{k_{2}}^{\left(q_{2}\right)} \cdots A_{k_{s-1} k_{s}} C_{k_{s}}^{\left(q_{s}\right)} x
$$

Note that the (resolvent of the) operator $A_{m}$ can appear here only at the first place, $k_{1}$. By the above considerations, the other operators denoted by the letter $C$ map any nonnegative nonzero element into a quasi-interior nonnegative element in the corresponding space, and the operators denoted by the letter $A$ map a quasiinterior element into a nonnegative nonzero element. Since the first operator either $\left(k_{1} \neq m\right)$ has the above mentioned property or (in the case $k_{1}=m$ ) is certainly invertible, the above product does not vanish. The proof is complete.

Remark. Observe that the proof of Theorem 2 in the matrix case (for the purposes of Corollary 2) simplifies considerably: then the matrices $C$ are, with the possible exception of $\left(R-A_{m}\right)^{-1}$, all (entrywise, i.e. strictly) positive, the matrices $A$ are all nonnegative and nonzero, and the statement of Theorem 2 follows immediately.

Using the Frobenius normal form of a matrix, we obtain in our terminology (which slightly differs from that of Rothblum $[\underline{\mathrm{R}}$ )

Corollary 2 (The Rothblum Index Theorem, $[\underline{\mathrm{R}}$, Theorem 3.1(2)]). Let $A$ be a square nonnegative matrix in a Frobenius normal form having spectral radius $R$. Then the order of the pole $R$ for $A$ (i.e. the index of $R-A$ ) is the maximum of the cardinalities of the basic classes in any (weighted) chain.

Proof. We claim that an arbitrary weighted chain with the lowest possible weight is (clearly nonnegative and) nonzero. We shall use the notation of (1), and consider first the case when $R>0$. If all classes (i.e. the submatrices $A_{j}$ ) are irreducible, then Theorem 2 applies immediately. If there are matrices $A_{k}$ equal to the $1 \times 1$ zero matrix, and a weighted chain (2) with the lowest possible weight $w$ contains several $C$ 's corresponding to them, then they must have the form $1 / R(1 \times 1$ matrices $)$, and multiplication from the left and right by the corresponding nonzero $A$ 's will yield again nonzero (dyadic) products. Having established this, the proof of Theorem 2 applies again. Finally, if $R=0$, then every $A_{j}$ is a $1 \times 1$ zero matrix. Hence every residuum (projection) $P_{j}$ is the $1 \times 1$ unit matrix 1 , and every weighted chain has the form

$$
1 a_{k_{1} k_{2}} 1 \cdots 1 a_{k_{s-1} k_{s}} 1
$$

where all the $a$ 's are positive numbers. The meaning of the lowest possible weight then yields the statement of the Corollary.

\section{EXAMPLES}

The treatment of the following example from [HRS] will show how effectively Theorem 1 can be applied in the relatively simple case of matrices of small sizes, when the pole is not necessarily the spectral radius.

Example 1. Let $z_{0}:=0$ and

$$
A:=\left(\begin{array}{lll}
0 & 0 & 0 \\
1 & 1 & 0 \\
1 & 1 & 0
\end{array}\right)
$$


Considering the entries as operators, the orders clearly are $\operatorname{ord}\left(0, A_{1}\right)=\operatorname{ord}\left(0, A_{3}\right)$ $=1$, and $\operatorname{ord}\left(0, A_{2}\right)=0$. The leading coefficients of the resolvents are (in the natural order) $1,-1,1$. The two admissible weighted chains from 3 to 1 are 1 and -1 , hence the $(3,1,-2)$-sum is equal to 0 . The $(1,1,-1)$-sum is 1 , and Theorem 1 shows that $\operatorname{ord}(0, A)=1$.

Example 2. Applying the notation from the beginning of Section 2, assume that all the spaces $X_{1}, \ldots, X_{m}$ are equal to a fixed Banach space $Y$, all the operators $A_{1}, \ldots, A_{m}$ on the diagonal are equal to an operator $B$ acting in $Y$, all the operators $A_{21}=\cdots=A_{m, m-1}$ are equal to an operator $T$ commuting with $B$, and that all other operator entries in $A$ are 0 . Assume further that $z_{0}$ is a pole of exact order $p \geq 1$ for the resolvent of the operator $B$. We claim that the order of the pole $z_{0}$ for the resolvent of the matrix operator $A$ is at most $p+m-1$, and is exactly $p+m-1$ if $T^{m-1} C^{(-p)} \neq 0$, where $C^{(-p)}$ denotes the leading coefficient operator in the Laurent expansion of the resolvent of $B$ around $z_{0}$. The last condition holds, e.g. if $T=I$, the identity operator in $Y$.

Indeed, any weighted chain of length $s$ with weight $w:=q_{1}+\cdots+q_{s}$ has, due to commutativity, the following form:

$$
T^{s-1} C^{\left(q_{1}\right)} \cdots C^{\left(q_{s}\right)},
$$

where the operators $C$ are the corresponding coefficients in the Laurent expansion of the resolvent of $B$ around $z_{0}$. From the general relations among these coefficients (see, e.g., [T, 5.8]) we know that the above product of the $C$ 's is zero if there are both nonnegative and negative superscripts, and yields $C$ with a positive superscript (times perhaps -1) if and only if every superscript is nonnegative. Further, if $q$ and $r$ are both negative superscripts, then

$$
C^{(q)} C^{(r)}=C^{(q+r+1)} .
$$

Hence, in this last case, the above weighted chain can be written as

$$
T^{s-1} C^{(w+s-1)} .
$$

By assumption, the lowest possible superscript is $-p$. Hence for the lowest possible weight $w(s)$ for the length $s$ we obtain

$$
w(s)=-p-s+1 .
$$

$w(s)$ assumes its smallest value when $s=m$; hence the order of the pole for the resolvent of $A$ is at most $p+m-1$ as stated. Any weighted chain (of length $m$ ) with the lowest possible weight $w(m)$ can be written as $T^{m-1} C^{(-p)}$. If it is a nonzero operator, so is any positive multiple of it. In this case the order of the pole $z_{0}$ will be exactly $p+m-1$.

Note that our argument shows that, more generally, if $s$ is the largest positive integer for which $T^{s-1} C^{(-p)} \neq 0$, then the order of the pole $z_{0}$ for $A$ is exactly $p+s-1$.

Finally note that the case $T=I$ may be called the case of an "operator Jordan block" of size $m$ with the operator $B$ in the diagonal positions. Clearly, if $\operatorname{dim} Y=1$ and $B=z_{0} I$ in the space $Y$, we obtain the usual (scalar) Jordan block of size $m$, and the order of the pole $z_{0}$ is $m$.

Example 3. Assume again in the notation from the beginning of Section 2 that all the Banach spaces $X_{1}, \ldots, X_{m}$ are equal to a fixed $l^{p}$ space $(1 \leq p \leq \infty)$, and 
the operators $A_{j}$ on the diagonal are multiplication operators, i.e. (representable by) diagonal infinite matrices

$$
A_{j}:=\operatorname{diag}\left[a_{j 1}, a_{j 2}, a_{j 3}, \ldots\right], \quad \max \left[\left|a_{j k}\right| ; k=1,2, \ldots\right]<\infty .
$$

For simplicity's sake we shall assume that all the complex numbers $a_{j k}$ are isolated points in the set $\left\{a_{j k} ; k=1,2, \ldots\right\}$ (their "multiplicity" may be infinite). Then $a_{j k}$ is a pole of order 1 for the resolvent of $A_{j}$ with corresponding leading coefficient

$$
P_{j k}:=C_{j}^{(-1)}=\operatorname{diag}[0, \ldots, 0,1,0, \ldots, 0,1,0, \ldots],
$$

where we find 1 exactly at the positions where we find the given $a_{j k}$ in the diagonal of $A_{j}$. Finally, we assume that in the subdiagonal there is the identity $I$ at each position, whereas all the other entries are 0 :

$$
A:=\left(\begin{array}{ccccc}
A_{1} & 0 & 0 & \ldots & 0 \\
I & A_{2} & 0 & \ldots & 0 \\
0 & I & A_{3} & \ldots & 0 \\
\ldots & \ldots & & & \\
0 & 0 & 0 & I & A_{m}
\end{array}\right) .
$$

Then it is clear that every $a_{j k}(j=1, \ldots, m ; k=1,2, \ldots)$ is a pole of order at least 1 of $A$. We claim that for a given $z_{0}:=a_{j_{0} k_{0}}$ the exact order of the pole $z_{0}$ for the resolvent of $A$ is

$$
\operatorname{ord}\left(z_{0}, A\right)=M:=\max _{1 \leq k<\infty} \operatorname{card}\left[j \in\{1, \ldots, m\} ; a_{j k}=z_{0}\right],
$$

where $\operatorname{card}[\cdot]$ denotes the cardinality of the set $\cdot$. At first note that for each $j \in$ $\{1, \ldots, m\}$ the resolvent operator $\left(z-A_{j}\right)^{-1}$ exists in a punctured neighborhood of $z_{0}$, and is a diagonal matrix:

$$
\left(z-A_{j}\right)^{-1}=\operatorname{diag}\left[\left(z-a_{j k}\right)^{-1} ; k=1,2, \ldots\right] .
$$

By [T, 5.8], we obtain for the 0th coefficient of the corresponding Laurent expansion around $z_{0}$ :

$$
C_{j}^{(0)}=(2 \pi i)^{-1} \int\left(z-z_{0}\right)^{-1}\left(z-A_{j}\right)^{-1} d z,
$$

where the integration path is a sufficiently small contour around the point $z_{0}$. It is clear that each $C_{j}^{(0)}$ is a diagonal matrix.

Pick now a maximizing value of $k$ for $M$, say $k_{1}$, and denote all the values of $j \in\{1, \ldots, m\}$ satisfying $a_{j k_{1}}=z_{0}$ (in decreasing order) by $j_{1}>j_{2}>\cdots>j_{M}$. We shall show that the only conceivably nonzero weighted chain in the $\left(j_{1}, j_{M},-M\right)$ weighted sum is in fact nonzero. It has the form

$$
C_{j_{1}}^{(-1)} C_{j_{1}-1}^{(*)} \cdots C_{j_{M}}^{(-1)},
$$

where the superscripts $(*)$ are equal to -1 exactly at the subscripts $j_{h}$ and are equal to 0 otherwise. For each subscript $j$ not equal to any $j_{h}$, the $k_{1}$ th diagonal element of the resolvent $\left(z-A_{j}\right)^{-1}$ is $\left(z-a_{j k_{1}}\right)^{-1}$, where $a_{j k_{1}} \neq z_{0}$. Hence the $k_{1}$ th diagonal element of the coefficient operator $C_{j}^{(0)}$ is

$$
(2 \pi i)^{-1} \int\left(z-z_{0}\right)^{-1}\left(z-a_{j k_{1}}\right)^{-1} d z=\left(z_{0}-a_{j k_{1}}\right)^{-1} \neq 0,
$$


where the integral is taken along a small contour around the point $z_{0}$. Hence the $k_{1}$ th diagonal element of the operator (3) is certainly nonzero. It follows that $\operatorname{ord}\left(z_{0}, A\right) \geq M$.

On the other hand, for every pair $(g, j)$ satisfying $m \geq g \geq j \geq 1$, the $(g, j,-M-1)$-sum is the operator 0 . Indeed, any weighted chain in the above sum has the form

$$
C_{g}^{\left(q_{1}\right)} C_{g-1}^{\left(q_{2}\right)} \cdots C_{j}^{\left(q_{s}\right)}, \quad q_{1}+\cdots+q_{s}=-M-1 .
$$

All the factor operators are diagonal matrices, and at least $M+1$ of them must be the corresponding spectral projections. By the definition of the number $M$, for each diagonal position there are at most $M$ spectral projections, i.e. operators of the form $C_{d}^{(-1)}$, for which the corresponding diagonal element is nonzero. Hence the product is the operator 0 , and $\operatorname{ord}\left(z_{0}, A\right)=M$.

Example 4. By slightly modifying a special case of Example 3, consider the following lower triangular operator matrix:

$$
A:=\left(\begin{array}{ccc}
I & 0 & 0 \\
I & 2 I & 0 \\
I & I & I
\end{array}\right)
$$

The number $z_{0}:=1$ is clearly a pole of order at least 1 of the resolvent of $A$, for, e.g., the $(1,1,-1)$-sum is not the operator 0 . On the other hand, the $(3,1,-2)$-sum is the sum of the following two weighted chains:

$$
C_{3}^{(-1)} C_{1}^{(-1)}+C_{3}^{(-1)} C_{2}^{(0)} C_{1}^{(-1)}=I-I=0 .
$$

Here the coefficient $C_{2}^{(0)}$ is calculated according to [T] 5.8], and is equal to the contour integral around the point 1

$$
(2 \pi i)^{-1} \int(z-1)^{-1}(z-2 I)^{-1} d z=-I .
$$

Hence the order of the pole 1 is equal to 1 .

Example 5. In the notation from the beginning of Section 2, let

$$
A:=\left(\begin{array}{cc}
A_{1} & 0 \\
A_{21} & A_{2}
\end{array}\right),
$$

and assume that all the operator entries are compact operators between their respective spaces. Then $A$ is also compact, and for the spectra $s(\cdot)$ we have $(\mathrm{cf}$. [N] or $[\mathrm{H}])$ :

$$
s(A) \cup s\left(A_{1}\right)=s\left(A_{1}\right) \cup s\left(A_{2}\right) .
$$

Hence any nonzero complex number is a pole of the resolvent of $A$ if and only if it is a pole of either $A_{1}$ or $A_{2}$. Assuming that $z_{0}$ is a pole for both $A_{1}$ and $A_{2}$ of orders $p_{1}>0$ and $p_{2}>0$, respectively, set

$$
-p_{21}:=\min \left\{w ; \sum_{q_{2}+q_{1}=w} C_{2}^{\left(q_{2}\right)} A_{21} C_{1}^{\left(q_{1}\right)} \neq 0\right\} .
$$

Then, by Theorem 1 , the order of the pole $z_{0}$ for $A$ is

$$
\max \left\{p_{1}, p_{2}, p_{21}\right\} .
$$




\section{ACKNOWLEDGEMENT}

The authors wish to thank a referee for his/her constructive remarks.

\section{REFERENCES}

[AB] C.Aliprantis and O.Burkinshaw, Positive operators, Academic Press, Orlando, 1985. MR 87h:47086

[BCN] R.Bru, J.J.Climent and M.Neumann, On the index of block upper triangular matrices, SIAM J. Matrix Anal. Appl., 16 (1995), 436-447. MR 96i:15011

$[\mathrm{FH}]$ S.Friedland and D.Hershkowitz, The rank of powers of matrices in a block triangular form, Linear Alg. Appl., 107 (1988), 17-22. MR 89h:15003

$[\mathrm{H}] \quad$ G. Hengstberger, Spectral properties of operator matrices, Tübinger Berichte zur Funktionalanalysis, 1 (1991/92), 65-72.

[HRS] D. Hershkowitz, U.G.Rothblum and H.Schneider, The combinatorial structure of the generalized nullspace of a block triangular matrix, Linear Alg. Appl., 116 (1989), 9-26. MR 90a:15026

[JLV] R-J. Jang-Lewis and H.D.Victory,Jr., On the ideal structure of positive, eventually compact linear operators on Banach lattices, Pacific J. Math., 157 (1993), 57-85. MR 93m:47042

[MR] C. D. Meyer and N.J.Rose, The index and the Drazin inverse of block triangular matrices, SIAM J. Appl. Math., 33 (1977), 1-7. MR 57:345

[N] R.Nagel, Towards a "matrix theory" for unbounded operator matrices, Math. Zeitschr., 201 (1989), 57-68. MR 90c:47004

[R] U.G.Rothblum, Algebraic eigenspaces of nonnegative matrices, Linear Alg. Appl., 12 (1975), 281-292. MR 53:8100

[SBL] H.H.Schaefer, Banach lattices and positive operators, Springer, Berlin, 1974. MR 54:11023

[ST] H.H.Schaefer, Topological vector spaces, 3rd print, Springer, Berlin, 1971. MR 49:7722

[T] A.E.Taylor, Introduction to functional analysis, Wiley, New York, 1958. MR 20:5411]

Department of Mathematics, Technical University Berlin, Sekr. MA 6-4, Strasse Des 17. Juni 135, D-10623 Berlin, Germany

E-mail address: foerster@math.tu-berlin.de

Department of Analysis, Institute of Mathematics, Technical University Budapest, H-1521 Budapest, Hungary

E-mail address: bnagy@math.bme.hu 